Bruno Laviolle ${ }^{13}$, Jean-Daniel Lelievre ${ }^{14}$, Jacques Morel ${ }^{15}$, Stéphanie Nguyen ${ }^{16}$, Jean-Philippe Spano ${ }^{17}$, Benjamin Terrier ${ }^{18}$, Anne Thiebaut ${ }^{19}$, Jean-Francois Viallard ${ }^{20}$, François Vrtovsnik ${ }^{21}$, Xavier de Lamballerie ${ }^{22}$ and Odile Launay ${ }^{2,23}$

${ }^{1}$ Department of Infectious and Tropical Diseases, INSERM U1047, Centre Hospitalier Universitaire (CHU) Nìmes, University of Montpellier, Nìmes, France. ${ }^{2}$ INSERM, French Clinical Research Infrastructure Network, Réseau Innovative Clinical Research in Vaccinology, Paris, France. ${ }^{3}$ Institut de Santé Publique d'Epidémiologie et de Développement, INSERM, Bordeaux Population Health Research Center, UMR 1219, Centre d'Investigation Clinique-Epidémiologie Clinique 1401, University of Bordeaux, Bordeaux, France. ${ }^{4}$ Service d'Information Médicale, CHU de Bordeaux, Pôle de Santé Publique, Bordeaux, France. ${ }^{5}$ Service Immunologie Biologique, Paris-Centre de Recherche Cardiovasculaire, INSERM U970, Assistance Publique-Hôpitaux de Paris (APHP), Hôpital Européen Georges Pompidou, Université de Paris, Paris, France. ${ }^{6}$ Fédération des Centre de Ressources Biologiques-Plateforme de Ressources Biologiques APHP, Centre-Université de Paris, Centre de Ressources Biologique Cochin, Hôpital Cochin, Paris, France. ${ }^{7}$ Service de Transplantation Rénale, Pitié Salpêtrière, APHP, Sorbonne Université, Paris, France. ${ }^{8}$ Centre Leon Berard UNICANCER et Université Lyon I, Lyon, France. ${ }^{9}$ Service de Néphrologie-Immunologie clinique, CHU Nantes, Nantes, France. ${ }^{10}$ Centre d'Investigation Clinique (CIC) Cochin Pasteur, APHP, Hôpital Cochin, Paris, France. ${ }^{11}$ Centre de Recherche en Transplantation et Immunologie-INSERM U1064, Service de Neurologie, CIC 1413, Université de Nantes, CHU Nantes, Nantes, France. ${ }^{12}$ Hospices Civils de Lyon, Université Claude Bernard Lyon 1, Association Française d'Etudes et de Recherche de l'Obésité, French Clinical Research Infrastructure Network-French Obesity Research Centre of Excellence Network, Nantes, France. ${ }^{13}$ University of Rennes, CHU Rennes, INSERM, CIC 1414, Rennes, France. ${ }^{14}$ Vaccine Research Institute, INSERM and APHP, Hôpital H. Mondor, Créteil, France. ${ }^{15}$ Rheumatology Department, $\mathrm{CHU}$ and University of Montpellier, Montpellier, France. ${ }^{16} \mathrm{APHP}-$ Sorbonne
Université, INSERM U1135, CNRS ERL 8255, Centre d'Immunologie et des Maladies Infectieuses-Paris, Paris, France. ${ }^{17}$ APHP-Sorbonne Université, Institut Pierre Louis d'Epidemiologie et de Santé Publique INSERM 1136, Paris, France. ${ }^{18}$ Service de Médecine Interne, Hôpital Cochin, APHP, Paris, France. ${ }^{19}$ Département d'Hématologie, CHU Grenoble Alpes, Grenoble, France. ${ }^{20}$ Université de Bordeaux, Hôpital Haut-Lévêque, Bordeaux, France. ${ }^{21}$ Service de Néphrologie, Hôpital Bichat-Claude Bernard, APHP, Departement Hospitalo-Universitaire Fire, Université de Paris, Paris, France. ${ }^{22}$ Unité des Virus Émergents, Aix-Marseille Université, Institut de Recherche pour le Développement 190, INSERM 1207, Institut Hospitalo-Universitaire Méditerranée Infection, Marseille, France. ${ }^{23}$ INSERM, CIC Cochin Pasteur, Assistance Publique Hôpitaux de Paris, Hôpital Cochin, Université de Paris, Sorbonne Paris Cité, Paris, France. ${ }^{24}$ These authors contributed equally: Paul Loubet, Linda Wittkop.

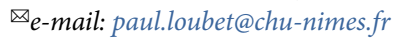

Published online: 12 July 2021

https://doi.org/10.1038/s41591-021-01435-1

References

1. Chavarot, N. et al. Transplantation https://doi.org/10.1097/ TP.0000000000003784 (2021).

2. Rozen-Zvi, B. et al. Clin. Microbiol. Infect. https://doi. org/10.1016/j.cmi.2021.04.028 (2021).

3. Boyarsky, B.J. et al. J. Am. Med. Assoc. https://doi.org/10.1001/ jama.2021.7489 (2021)

4. Benotmane, I. et al. Kidney Int. 99, 1487-1489 (2021).

5. Grupper, A. et al. Clin. J. Am. Soc. Nephrol. https://doi. org/10.2215/CJN.03500321 (2021).

6. Agur, T. et al. Nephrol. Dial. Transplant. https://doi.org/10.1093/ ndt/gfab155 (2021).

7. Monin, L. et al. Lancet Oncol. 22, 765-778 (2021).

8. Barrière, J. et al. Ann. Oncol. https://doi.org/10.1016/j. annonc.2021.04.019 (2021).

9. Kennedy, N. A. et al. Gut https://doi.org/10.1136/ gutjnl-2021-324789 (2021).

\section{Acknowledgements}

We thank the patients of the ANRS0001S COV-POPART Cohort; the scientific committee of the ANRS0001S COV-POPART Study Group (P. Loubet, O. Launay, L. Wittkop, E. Tartour, B. Parfait, B. Barrou, J.-Y. Blay, M. Hourmant, M. Lachâtre, D.-A. Laplaud, M. Laville, B. Laviolle, J.-D. Lelievre, J. Morel, S. Nguyen, J.-P. Spano, B. Terrier, A. Thiebaut, J.-F. Viallard, F. Vrtovsnik, X. de Lamballerie, M. Vialemaringe, L. Esterle, J. Longobardi, A. Levier, S. Le Mestre, S. Lancrey-Javal, I. Ortega-Perez, A. Bouakane, A. Diallo, L. Meyer, P. Vanhems, A. Trouiller,
B. Thevenin Lemoine, E. Buleux, E. Plassart, A.-S. Joly, I. Sartori, J.-F. Thebaut, A. Huet, N. André, L. Vallet, A. de Guerra Remi Slama, D. Deplanque and P. Rossignol); the steering committee of the ANRS0001S COV-POPART Study Group (P. Loubet, O. Launay, L. Wittkop, E. Tartour, X. de Lamballerie, L. Esterle, J. Longobardi, S. Kamal, A.Levier, S. Le Mestre, S. Lancrey-Javal, I. Ortega-Perez, A. Bouakane, B. Parfait, S. Circosta, L.-Victorien Vieillard and A. Boston); the Investigation centers of the ANRS0001S COV-POPART study (CHU Saint Etienne, CHU Besançon, Hôpital Foch, CHU Henri Mondor (APHP), CHU La Pitié-Salpétrière (APHP), CHU Angers, CHU Nantes, $\mathrm{CH}$ La Roche sur Yon, CHU Nice, CHU Caen, CHU Clermont-Ferrand, Hospices Civiles de Lyon, $\mathrm{CHU}$ Cochin (APHP), CHU Nîmes, Centre Léon Bérard, CHU Bichat (APHP), CHU Saint-Louis (APHP), CHU Tours, CHU Strasbourg, CHU Montpellier, CHU Dijon, CHU Saint-Antoine (APHP), CHU Bordeaux, CHU Toulouse, CHU Lille, Marseille (Assistance Publique-Hôpitaux de Marseille), Hôpital Saint Joseph (Marseille), CHU Grenoble, CHU Nancy, CHU Rouen, CHU Brest, CHU Limoges, CHU Rennes, CHU HEGP (APHP), Clinique Bordeaux Nord, Hôpital Européen de Marseille and Institut Gustave Roussy); and the scientific societies (Société Francophone de Sclérose En Plaques, Fondation ARSEP, Société Francophone de Greffe de Moelle et de Thérapie Cellulaire (SFGM-TC), Société Francophone de Transplantation (SFT), Société Française de Rhumatologie (SFR), Société Française de Néphrologie Dialyze et Transplantation (SFNDT), Société Francophone du diabète, de la Société Française d'endocrinologie, de l'AFERO (association Française d'Études et de recherche pour l'Obésité) et de la Société Française de Nutrition (SFN), FORCE, Société Française Oncologie Médicale, Fédération Francophone de Cancérologie, Société Française du Cancer, Société Française d'Immunologie (SFI), Société de Pathologie Infectieuse de Langue Française (SPILF), Unicancer, LYRICAN (INCA-INSERM DGOS), EURACAN (European Commission)). Funding was received from ANRS | Emerging Infectious Diseases, Ministère des Solidarités et de la Santé and Ministère de l'Enseignement Supérieur, de la Recherche et de l'Innovation.

\section{Competing interests}

P.L. has received personal fees as a speaker or consultant from Pfizer and Astrazeneca. J.M. has received honoraria as a speaker or consultant of less than $€ 8,000$ from Abbvie, Biogen, Bristol Myers Squibb, Boerhinger Ingelheim, Galapagos, GlaxoSmithKline, Fresenius Kabi, Lilly, Mylan, Novartis, Pfizer and Sanofi, and grants (outside the submitted work) from Lilly and Novartis. J.P.S. has received personal fees as a speaker or consultant from Pfizer and Astrazeneca. O.L. has received personal fees from Sanofi Pasteur; grants, personal fees and non-financial support from Pfizer, Janssen and Sanofi Pasteur-Merck Sharp \& Dohme; and grants and non-financial support from GlaxoSmithKline.

\title{
Fractionation of COVID-19 vaccine doses could extend limited supplies and reduce mortality
}

To the Editor-COVID-19 continues to pose a major threat to public health. Public-health and social measures have been implemented to control transmission, but they are emergency measures that are difficult to sustain in the longer term.
There are now 15 vaccines against COVID19 being used worldwide. However, shortages in the supply of vaccines have been a particular problem for low-income countries, which have collectively received only $0.2 \%$ of all vaccines delivered worldwide for approximately $10 \%$ of the world's population. Fractionation of vaccine doses (Fig. 1) is a potential solution to this global shortage of vaccines that has not been given sufficient attention and consideration. 
a

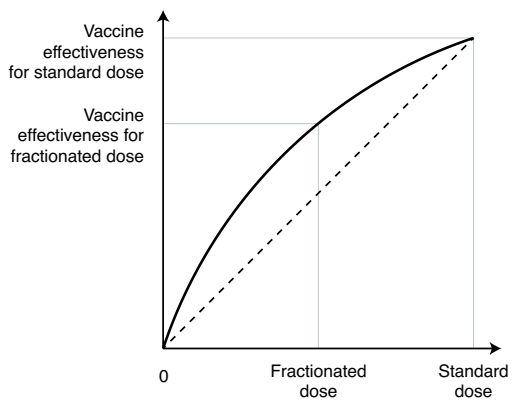

b

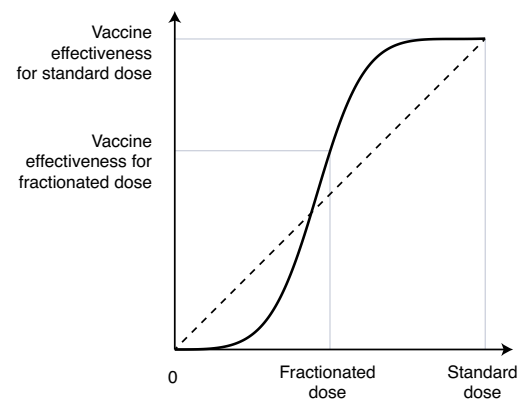

Fig. 1 | Rationale for dose fractionation to make more-effective use of limited supplies of vaccine antigen. a, Solid line indicates a scenario in which there is a concave relationship between vaccine efficacy (vertical axis) and dosage (horizontal axis), whereby a fractionated dose (e.g., approximately half of a standard dose) could provide considerably more than half the effectiveness of the standard dose; the dashed diagonal line is included for reference. If the concave relationship holds, it indicates that providing half doses to a certain number of people could provide a greater level of population immunity than would providing standard doses to half as many people. For reference, the population immunity conferred by vaccination could be estimated via the vaccination coverage multiplied by vaccination effectiveness. $\mathbf{b}$, Another scenario in which very low fractionated doses might not provide any clinical benefit, but fractionation would still provide population benefits above a certain threshold. Here, the solid line crosses the diagonal dashed line at doses approaching half a standard dose, and at the fractionated dose indicated (approximately half a standard dose), there would be an advantage for population immunity to use half doses.

In 2015, emergency vaccination against yellow fever was needed to mitigate epidemics in Angola and the Democratic Republic of Congo. However, there were limited supplies of the vaccine and there was a 6-month minimum manufacturing process $^{1}$. The World Health Organization's Strategic Advisory Group of Experts on Immunization reviewed evidence on the immunogenicity and safety of fractional dosing of vaccines against yellow fever and recommended dose fractionation down to one fifth of the standard dose ${ }^{2}$. This was put into practice in Angola and the Democratic Republic of Congo in 2016, with millions vaccinated with the fractional dose, and was subsequently put into practice in Brazil in 2017-2018 (ref. ${ }^{3}$ ). Fractional dosing was predicted to substantially reduce population infection attack rates and save lives $^{1}$. Vaccine-dose fractionation has also been recommended by the World Health Organization for inactivated poliovirus vaccines and meningococcal conjugate vaccines as an alternative mass-vaccination strategy, particularly when vaccine shortages occur during outbreaks in resource-limited locations.

Dose-finding studies indicate that fractional doses of mRNA vaccines could still elicit a robust immune response to COVID-192,3. In a non-randomized open-label phase $1 / 2$ trial of the BNT162b2 vaccine, doses as low as one third $(10 \mu \mathrm{g})$ of the full dose produced antibody and cellular immune responses comparable to those achieved with the full dose of $30 \mu \mathrm{g}$ (ref. $\left.{ }^{4}\right)$. Specifically, the geometric mean titer of neutralizing antibodies 21 days after the second vaccine dose was 166 for the group that received $10 \mu \mathrm{g}$, almost the same as the geometric mean titer of 161 for the group that received $30 \mu \mathrm{g}$, and 63 days after the second dose, these titers were 181 and 133, respectively ${ }^{4}$. For the mRNA-1273 vaccine, a dose of $25 \mu \mathrm{g}$ conferred geometric mean $\mathrm{PRNT}_{80}$ titers (the inverse of the concentration of serum needed to reduce the number of plaques by $80 \%$ in a plaque reduction neutralization test) of 340 at 14 days after the second dose, compared with a value of 654 for the group that received the standard dose of $100 \mu \mathrm{g}$ (ref. ${ }^{5}$ ). According to the model proposed by Khoury et al. ${ }^{6}$, if vaccine efficacy at the full dose is $95 \%$, a reduction in dose that led to as much as a halving in the post-vaccination geometric mean titer could still be in the range of 85-90\%. Although other components of the immune response may also contribute to efficacy, these dose-finding data are at least indicative of the potential for further exploration of fractionation as a dose-sparing strategy. Durability of responses after fractional doses should also be explored.

We are aware of only one trial reporting data on the clinical efficacy of a fractionated dosing strategy for COVID-19, which was a trial of the AZD1222 vaccine in the UK'
As part of a larger multicenter trial, Voysey et al. reported a vaccine efficacy of $90 \%$ (67-97\%) among a subgroup of participants who were primed with a half dose instead of a full dose, followed by a full-dose boost after a median of 12 weeks ${ }^{7}$. Although only a small number of participants were included in this subgroup, the lower bound of $67 \%$ for the efficacy estimate is very reassuring.

Concerns about the evolution of vaccine resistance have been posited as a potential drawback of dose-sparing strategies. However, vaccines that provide protection against clinical disease seem to also reduce transmission, which indicates that expanding partial vaccination coverage could reduce the incidence of infection. As described in a recent paper, lower prevalence should slow, not accelerate, the emergence and spread of new

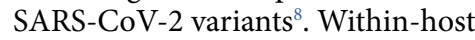
dynamics are unlikely to overcome these population-level effects. In contrast to some chronic infections, such as infection with human immunodeficiency virus, infection with SARS-CoV-2 in healthy people does not readily select for variants that escape the immune system. Such rapid selection has been reported only in immunocompromised people, who might have better access to vaccines if doses were fractionated in the general population. One other potential concern about fractionation would relate to vaccine hesitancy, if fractionated doses were viewed as inferior. However, the strategy of delaying the second dose of the vaccine applied in the UK and elsewhere has been well accepted as a strategy for providing at least partial protection to a greater number of people ${ }^{8}$.

In conclusion, fractionated doses could provide a feasible solution that extends limited supplies of vaccines against COVID-19, which is a major challenge for low- and middle-income countries. We identified several ongoing dose-finding studies on the ClinicalTrials.gov website that will provide important evidence to support fractionation policies. Although most dose-finding studies focus on immune responses with relatively small sample sizes, larger trials to estimate the efficacy of fractionated doses would also be worthwhile. The mRNA vaccines may be particularly suited for fractionation because of their higher levels of efficacy relative to those other vaccine types, while the favorable clinical-trial results on fractionation of the first dose of AZD1222 should encourage further investigation ${ }^{7}$. Even though some locations are reaching higher levels of vaccine coverage, many parts of the world with low vaccination rates could benefit from fractionation if it were determined 
to be an advantageous strategy. Of course, vaccination rates are restricted not only by antigen supply but also by the availability of vaccinators and clinical supplies, such as syringes. Nevertheless, antigen supply remains the greatest restriction for vaccines against COVID-19, and strategies that make the most of available antigen supply would maximize the number of lives saved by vaccination. More generally, in future pandemics, researchers and clinicians involved in vaccine development should consider identifying the appropriate vaccine dosage that can save the most lives for a limited amount of antigen, rather than just a dosage that balances efficacy and reactogenicity for the individual person.
${ }^{1}$ World Health Organization Collaborating Centre for Infectious Disease Epidemiology and Control, School of Public Health, Li Ka Shing Faculty of Medicine, The University of Hong Kong, Hong Kong Special Administrative Region, Hong Kong, China. ${ }^{2}$ Laboratory of Data Discovery for Health Limited, Hong Kong Science and Technology Park, New Territories, Hong Kong Special Administrative Region, Hong Kong, China. ${ }^{3}$ Department of Ecology and Evolution, University of Chicago, Chicago,

IL, USA.

$\bigotimes_{e-m a i l: b c o w l i n g @ h k u . h k}$

Published online: 5 July 2021

https://doi.org/10.1038/s41591-021-01440-4

References

1. Wu, J. T., Peak, C. M., Leung, G. M. \& Lipsitch, M. Lancet 388, 2904-2911 (2016)

2. World Health Organization. Vaccine 35, 5751-5752 (2017).

3. World Health Organization. Wkly. Epidemiol. Rec. 33, 365-380 (2019)
4. Sahin U. et al. Preprint at medRxiv https://doi.org/ 10.1101/2020.12.09.20245175 (2020)

5. Jackson, L. A. et al. N. Engl. J. Med. 383, 1920-1931 (2020).

6. Khoury, D.S. et al. Nat. Med. https://doi.org/10.1038/s41591-021 01377-8 (2021).

7. Voysey, M. et al. Lancet 397, 99-111 (2021).

8. Cobey, S., Larremore, D. B., Grad, Y. H. \& Lipsitch, M. Nat. Rev. Immunol. 21, 330-335 (2021).

\section{Acknowledgements}

B.J.C. is supported by a fellowship award from the Research Grants Council of the Hong Kong Special Administrative Region (Project No. HKU SRFS2021-7S03).

\section{Author contributions}

B.J.C. conceived of this Comment; B.J.C. wrote the original draft of the manuscript with contributions from all authors; and all authors reviewed and approved the final version of the manuscript.

\section{Competing interests}

B.J.C. has consulted for GlaxoSmithKline, Sanofi Pasteur, AstraZeneca, Roche and Moderna.

\section{Has SARS-CoV-2 reached peak fitness?}

To the Editor-For the first time in the history of medicine, the evolution of a virus can be observed 'as it happens'. Throughout this, molecular tools of unprecedented accuracy are also assisting by providing an extraordinarily detailed picture of the interactions between the virus and the immune system of its human host.

In the initial period of the COVID-19 pandemic, the coronavirus SARS-CoV-2 used the plasticity of its genome to obvious advantage, increasing its transmissibility with the early appearance of the D614G mutation that led to a $20 \%$ increased infectiousness and that rapidly became the global dominant strain ${ }^{1}$. The next major jump in transmissibility appeared in late 2020 with the B.1.1.7 variant, which had an approximate $50 \%$ increase in transmissibility beyond that of $\mathrm{D} 614 \mathrm{G}^{2}$. The B.1.617.2 'India' variant has recently become prominent in many countries, and although the magnitude is uncertain, it represents another incremental increase in contagiousness beyond that of D614G and possibly that of B.1.1.7 ${ }^{3}$.

In parallel, there has been evolution of variants with properties that allow them to evade the immune system. Thus far, the B.1.351 variant has been demonstrated to have the greatest ability to escape the immune system, although not at a level that has substantially diminished the protection provided by vaccines.
The P.1 variant also has considerable ability to evade the immune response. Although the immunoevasion of D614G and B.1.1.7 is fairly modest, it appears that B.1.617.2 has some features that combine enhanced transmissibility and immunoevasion. Some of the recent enhancement in the ability to escape the immune system is 'tagged' to mutations that alter the $\mathrm{N}$-terminal domain rather than the spike protein's receptor-binding domain, where the early, functionally important alterations were prevalent ${ }^{3}$.

This progression of variants demonstrates the virus's drive for heightened fitness, the natural selection of mutations and strains that make it more likely to find hosts and are further facilitated by sidestepping the immune response, even allowing repeat infections of people who previously had COVID-19. We emphasize, however, that enhanced transmissibility, rather than immunoevasion or greater lethality, would be considered the most potent path for the virus to become more fit and viable.

Indeed, more-fit variants can be expected to emerge over time (the occurrence of which will need to be monitored meticulously, as these pose a potential public health threat), but we believe that these will not continue to emerge indefinitely: nothing is infinite in nature, and eventually the virus will reach its form of 'maximum transmission'. After then, new variants will provide no further advantage in infectivity. The virus will thus stabilize and this 'final' variant will prevail and become the dominant strain, experiencing only occasional, minimal variations.

By homology, we can imagine that the same took place when some very contagious RNA viruses (e.g., measles virus) spilled over in humans: in the early stages of the epidemic, the virus was probably unstable and less transmissible than it is now; then-once the most contagious phenotype was reached-the measles virus stabilized. We note that the inevitable outcome of this strategy for all RNA viruses that have developed high contagiousness (beyond measles virus, we could name, for instance, the viral agents that cause hepatitis A, poliomyelitis, mumps and rubella) is the lack of molecular structures that allow the virus to 'dodge' the immune response of the recovered host. Why is that? To make a long story short, by the time these viruses are attacked by the adaptive immunity of their new host, they have no immediate advantage in evading it, because they have probably already spread to another susceptible host, where replication and survival are ensured ${ }^{4}$.

Notably, the natural history of the SARS-CoV-2 virus-host interplay is now being deeply altered-an unprecedented 